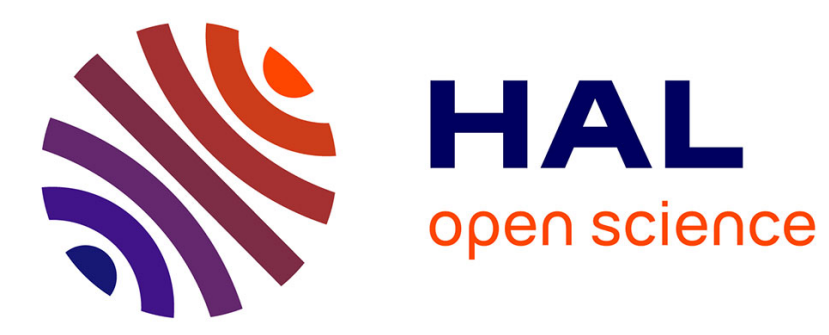

\title{
Lagrange multipliers in infinite dimensional spaces, examples of application
}

\author{
A. Bersani, F. Dell'Isola, Pierre Seppecher
}

\section{To cite this version:}

A. Bersani, F. Dell'Isola, Pierre Seppecher. Lagrange multipliers in infinite dimensional spaces, examples of application. 2019. hal-02269765

\section{HAL Id: hal-02269765 \\ https://hal.science/hal-02269765}

Preprint submitted on 23 Aug 2019

HAL is a multi-disciplinary open access archive for the deposit and dissemination of scientific research documents, whether they are published or not. The documents may come from teaching and research institutions in France or abroad, or from public or private research centers.
L'archive ouverte pluridisciplinaire HAL, est destinée au dépôt et à la diffusion de documents scientifiques de niveau recherche, publiés ou non, émanant des établissements d'enseignement et de recherche français ou étrangers, des laboratoires publics ou privés. 


\title{
Lagrange multipliers in infinite dimensional spaces, examples of application
}

\author{
A. Bersani, F. dell'Isola, P. Seppecher
}

\section{Synonyms}

Infinite-dimensional constrained mechanical systems

\section{Definitions}

The Lagrange multipliers method is used in Mathematical Analysis, in Mechanics, in Economics and in several other fields, to deal with the search of the global maximum or minimum of a function, in presence of a constraint. The usual technique, applied to the case of finite-dimensional systems, transforms the constrained optimization problem into an unconstrained one, by means of the introduction of one or more multipliers and of a suitable Lagrangian function,to be optimized. In Mechanics, several optimization problems can be applied to infinite-dimensional systems. Lagrange multipliers method can be applied also to these cases.

\section{Introduction}

In this entry we show that the theorem of Lagrange multipliers in infinite dimensional systems [1] can be a very powerful tool for dealing with constrained problems also in infinite dimensional spaces. This tool is powerful but must be used carefully. As penalization is often invoked as an intuitive and numerically efficient approach to constrained problems, we also show that the penalization approach may present the same drawbacks as a rough application of Lagrange multipliers method does. To that aim we describe two examples issued from continuum mechanics [2].

The first one goes back to Lagrange himself [3]: an incompressible fluid, or an incompressible linear elastic material is a material whose displacement field is constrained to be divergence-free. We show how easily the Lagrange multipliers method gives the system of balance equations and introduces the pressure as a supplementary unknown of the problem. The approach through penalization gives the same balance equations and the displacement field becomes divergence-free at the limit only. It is not very difficult to understand 
why the pressure which is not independent from the displacement until the limit, becomes an independent quantity at the limit. Hence both approaches are efficient for understanding what an incompressible material is and how it behaves.

The second example requires us to be more cautious. It consists in considering that second gradient materials are nothing else than micromorphic materials subjected to the constraint that the micro-deformation coincides with the gradient of the displacement field. We show that a careful application of the Lagrange multipliers method leads to the correct system of equilibrium equations and we warn against its possible erroneous applications. We show that the penalization method, when over-interpreted, may lead to the same errors: one cannot infer from the admissible boundary conditions for a micromorphic material what can be the boundary conditions for the constrained model, that is for the second gradient model. The discrepancy between results obtained by studying directly the critical points of a second gradient energy and those obtained by a rough limit of micromorphic models has led to some confusion which still survives.

\section{Incompressible materials}

Consider an elastic material contained in a bounded Lipschitz domain $\Omega$. Its elastic energy density depends on the first gradient of the displacement field $u$. The global energy has the form ${ }^{1}$

$$
\mathcal{L}(u):=\int_{\Omega} \ell(u(x), \nabla u(x)) d \mathcal{H}^{3}
$$

The material is said to be linearly incompressible ${ }^{2}$ if $u$ satisfies almost everywhere in $\Omega$ the condition

$$
\operatorname{div}(u)=0 .
$$

Assuming a quadratic growth at infinity for $\mathcal{L}$ with respect to its second argument, the natural functional framework is the Sobolev space $H^{1}\left(\Omega, \mathbb{R}^{3}\right)$. In the sequel $L^{2}$ and $H^{1}$ stand for the Sobolev spaces $L^{2}\left(\Omega, \mathbb{R}^{3}\right)$ and $H^{1}\left(\Omega, \mathbb{R}^{3}\right)$.

The goal is to write the partial differential equation that any smooth critical point $u$ of the global energy must solve. We thus assume that both $u$ and $\ell$ are of class $C^{2}$.

\subsection{Application of Lagrange Multiplier Theorem}

It is well known that the divergence operator is surjective from $H^{1}$ onto $L^{2}$. Indeed, for any $f \in L^{2}$, it is enough to remind that the Dirichlet problem $\Delta v=f$ on $\Omega$ with $v=0$ on the boundary admits a solution $\bar{v}$ and thus that

\footnotetext{
${ }^{1}$ Here $\mathcal{H}^{3}$ stands for the Lebesgue measure on $\mathbb{R}^{3}$.

${ }^{2}$ Rubber is an example of an incompressible elastic material. Incompressibility is also of high importance in hydrodynamics: in that case $u$ must be interpreted as the velocity field and $\mathcal{L}(u)$ as the associated dissipation.
} 
$f=\operatorname{div}(\nabla \bar{v})$. Duality in the Hilbert space $L^{2}$ is trivial: Lagrange multiplier theorem states the existence of a $L^{2}$ function $-p$ such that any critical point $u$ of $\mathcal{L}$ under the constraint (1) satisfies

$$
\int_{\Omega}\left(\partial_{1} \ell \cdot h+\partial_{2} \ell \cdot \nabla h-p \cdot \operatorname{div}(h)\right) d \mathcal{H}^{3}=0 .
$$

or

$$
\int_{\Omega}\left(\partial_{1} \ell \cdot h+\left(\partial_{2} \ell-p I d\right) \cdot \nabla h\right) d \mathcal{H}^{3}=0
$$

for any smooth test function $h$. Here the partial derivatives of $\ell$ are taken at $(u(x), \nabla u(x))$.

Hence, in the sense of distributions on $\bar{\Omega}$,

$$
\partial_{1} \ell \mathcal{H}_{\mid \Omega}^{3}-\operatorname{div}\left(\left(\partial_{2} \ell-p I d\right) \mathcal{H}_{\mid \Omega}^{3}\right)=0
$$

Now let us use the divergence theorem ${ }^{3}$ :

$$
\operatorname{div}\left(\varphi \mathcal{H}_{\mid \Omega}^{3}\right)=\operatorname{div}(\varphi) \mathcal{H}_{\mid \Omega}^{3}-(n \cdot \varphi) \mathcal{H}_{\mid \partial \Omega}^{2}
$$

where $n$ stands for the outward normal to $\partial \Omega$ and $\mathcal{H}_{\mid \partial \Omega}^{2}$ stands for the restriction to the boundary $\partial \Omega$ of the two-dimensional Hausdorff measure. We get

$$
\left(\partial_{1} \ell-\operatorname{div}\left(\left(\partial_{2} \ell-p I d\right)\right) \mathcal{H}_{\mid \Omega}^{3}+n \cdot\left(\partial_{2} \ell-p I d\right) \mathcal{H}_{\mid \partial \Omega}^{2}=0 .\right.
$$

In mechanics the quantities $-\partial_{1} \ell$ and $\partial_{2} \ell-p I d$ are respectively denoted $f$ and $\sigma$ and interpreted as the bulk external force and the stress tensor. Using this notation, previous equation reads

$$
-(f+\operatorname{div}(\sigma)) \mathcal{H}_{\mid \Omega}^{3}+n \cdot \sigma \mathcal{H}_{\mid \partial \Omega}^{2}=0 .
$$

As the measures $\mathcal{H}_{\mid \Omega}^{3}, \mathcal{H}_{\mid \partial \Omega}^{2}$ are orthogonal (or mutually singular) [5], this equation splits in

$$
\begin{aligned}
& f+\operatorname{div}(\sigma)=0 \quad \mathcal{H}^{3} \text { a.e. in } \Omega \\
& n \cdot \sigma=0 \quad \mathcal{H}^{2} \text { a.e. on } \partial \Omega .
\end{aligned}
$$

We recover the standard equilibrium equations where the stress tensor $\sigma=$ $\partial_{2} \ell-p I d$ involves explicitly the Lagrange multiplier $-p$. Its opposite $p$ is interpreted as an unknown pressure which has to be determined together with the equilibrium displacement $u$ by using the equilibrium equations together with the constraint $\operatorname{div}(u)=0$.

\footnotetext{
${ }^{3}$ Indeed, for any $C^{1}$ function $\varphi$ and any smooth test function $\psi$, $\left\langle\operatorname{div}\left(\varphi \mathcal{H}_{\mid \Omega}^{3}\right), \psi\right\rangle=-\left\langle\varphi \mathcal{H}_{\mid \Omega}^{3}, \nabla \psi\right\rangle=-\int_{\Omega} \varphi \cdot \nabla \psi d \mathcal{H}^{3}=\int_{\Omega} \operatorname{div}(\varphi) \cdot \psi d \mathcal{H}^{3}-\int_{\partial \Omega}(n \cdot \varphi) \psi d \mathcal{H}^{2}$
} 


\subsection{Approach through penalization}

An intuitive way for dealing with incompressibility is to penalize the fact that $\operatorname{div}(u)$ does not vanish. Looking for minimizers of the total energy, one decides to look for a sequence of approximate minimizers by considering, instead of the original potential subjected to the constraint, the original Lagrangian to which one adds a penalization term: for instance

$$
\mathcal{L}_{\varepsilon}(u)=\int_{\Omega}\left(\ell(u(x), \nabla u(x))+\varepsilon^{-1}(\operatorname{div}(u))^{2}\right) d \mathcal{H}^{3} .
$$

This corresponds to a slightly compressible elastic material. Denoting $-p_{\varepsilon}:=$ $2 \varepsilon^{-1} \operatorname{div}(u)$, any critical $u$ satisfies, for any smooth test function $h$,

$$
\int_{\Omega}\left(\partial_{1} \ell \cdot h+\partial_{2} \ell \cdot \nabla h-p_{\varepsilon} \operatorname{div}(h)\right) d \mathcal{H}^{3}
$$

This equation is identical to (2). It then leads to the same system of equilibrium equations. The difference is that now the stress tensor is directly related to $u$ through the so-called constitutive equation $\sigma=\partial_{2} l+2 \varepsilon^{-1} \operatorname{div}(u) I d$. When passing to the limit $\varepsilon \rightarrow 0$, $\operatorname{div}(u)$ tends to zero, the system of equilibrium equations is preserved but the constitutive equation is partially lost as $\varepsilon^{-1} \operatorname{div}(u)$ is an undetermined form.

\section{Second gradient as constrained generalized model}

Consider an elastic material contained in a bounded simply connected domain $\Omega$ with piecewise $C^{1}$ boundary. In the sequel $L^{2}$ and $H^{s}$ stand for the Sobolev spaces $L^{2}\left(\Omega, \mathbb{R}^{3}\right)$ and $H^{s}\left(\Omega, \mathbb{R}^{3}\right)$.

The elastic material is said to be a second gradient material (or equivalently a strain-gradient one) if its elastic energy density depends on the gradient and the second gradient of the displacement field $u$. The global energy has the form

$$
\int_{\Omega} l(u(x), \nabla u(x), \nabla \nabla u(x)) d \mathcal{H}^{3}
$$

Such a model and particularly the associated boundary conditions present some difficulties of interpretation (see for instance $[9,10,11]$ ). That is why many researchers prefer to see this model as a special case of a generalized continuum [8].

An elastic material is said to be a micromorphic generalized continuum if its elastic energy density depends on an extra matrix valued kinematic quantity $v$ and on the first gradients of $u$ and $v$. The global elastic energy has the form

$$
\mathcal{L}(u, v):=\int_{\Omega} \ell(u(x), v(x), \nabla u(x), \nabla v(x)) d \mathcal{H}^{3}
$$

It is clear that, when such a model is constrained in such a way that $v$ has to coincide with the first gradient of $u$, that is if

$$
C(u, v):=v-\nabla u=0,
$$


then one recovers the previous second gradient model.

This indirect approach does coincide with the direct approach under the express condition that the Lagrange Multiplier Theorem is precisely respected. A rough application of Lagrange Multiplier Method may lead and have led to misunderstanding boundary conditions.

In the sequel we assume that the considered energies have quadratic growth at infinity so the Sobolev spaces $L^{2}\left(\Omega, \mathbb{R}^{3}\right), H^{s}\left(\Omega, \mathbb{R}^{3}\right)$ or $H^{s}\left(\Omega, \mathbb{R}^{3 \times 3}\right)$ are the natural framework. We simply denote $L^{2}$ and $H^{s}$ these spaces as the tensorial nature of the involved functions is clear from the context.

We are thus looking for smooth critical points of the functional $\mathcal{L}(u, v)$ defined on $H^{1} \times H^{1}$ under the constraint $C(u, v)=0$. It is obvious that this problem can be simplified by setting ${ }^{4} l(u, \eta, \xi):=\ell(u, \eta, \eta, \xi)$ and by looking for the critical points of

$$
L(u, v):=\int_{\Omega} l(u(x), \nabla u(x), \nabla v(x)) d \mathcal{H}^{3}
$$

under the same constraint.

\subsection{Application of Lagrange Multiplier Theorem}

As our functionals are defined on the Hilbert space $H^{1} \times H^{1}$, assumed to be differentiable, and as the constraint $C(u, v)=0$ is linear, it is enough for applying Lagrange Multiplier Theorem [1] to check that the range $F$ of $C$ is a Banach space and to identify its dual.

Actually $F$ coincides with the Hilbert space $H_{\text {curl }}$ which has been extensively studied $[4,6]$. This space is defined as the set of all functions in $L^{2}(\Omega)$ whose curl (in the sense of distributions on $\Omega$ ) belongs to $L^{2}(\Omega)$. As $\operatorname{curl}(\nabla u)=0$, $\operatorname{curl}(C(u, v))=\operatorname{curl}(v) \in L^{2}(\Omega)$ and so the inclusion $F \subset H_{\text {curl }}$ is obvious. For proving the converse inclusion, let us consider $w \in H_{\text {curl }}$ and let us use Helmholtz decomposition of $\operatorname{curl}(w) \in L^{2}$ (see theorem 4.2 of $[6]$ ) and write $\operatorname{curl}(w)=\nabla \phi+\operatorname{curl}(v)$ with $\phi \in H_{0}^{1}(\Omega)$ and $v \in H^{1}(\Omega)$. Obviously $\phi \in$ $H_{0}^{1}(\Omega)$ satisfies $\Delta \phi=0$ and thus, owing to the maximum principle, it vanishes. So $\operatorname{curl}(w-v)=0$ in the sense of distributions which is equivalent to the orthogonality of $w-v$ with the curl of every smooth test function that is with every divergence-free smooth function. Hence (see [4] page 217) $(w-v) \in L^{2}(\Omega)$ is a gradient: $w-v=\nabla u$ with $u \in H^{1}(\Omega)$. We have $w=v+\nabla u=C(-u, v) \in F$.

The simplest way for identifying the dual $F^{*}$ consists in taking advantage of the Hilbert structure of $H_{\text {curl }}$ and applying Riez Theorem: to any element $\Lambda$ of $F^{*}$ is associated a function $f_{\Lambda}$ in $H_{\text {curl }}$ such that, for any $w \in H_{\text {curl }}$, $\langle\Lambda, w\rangle=\int_{\Omega}\left(f_{\Lambda} \cdot w+\operatorname{curl}\left(f_{\Lambda}\right) \cdot \operatorname{curl}(w)\right) d \mathcal{H}^{3}$. As smooth functions on $\bar{\Omega}$ are dense (Theorem 2 p. 204 of [4]) and continuously embedded in $H_{\text {curl }}, \Lambda$ is completely determined by its values for such smooth functions $w$. For such

\footnotetext{
${ }^{4}$ Note that $\partial_{1} l=\partial_{1} \ell, \partial_{2} l=\partial_{2} \ell+\partial_{3} \ell$ and $\partial_{3} l=\partial_{4} \ell$ where $\partial_{i} f$ stands for the partial derivative of a function $f$ with respect to its $i$-th variable. The densities $l$ and $\ell$ are assumed to be of class $C^{1}$.
} 
functions, one can integrate by parts in the sense of distributions on $\bar{\Omega}$ and get $\langle\Lambda, w\rangle=\left\langle f_{\Lambda}+\operatorname{curl}\left(\operatorname{curl}\left(f_{\Lambda}\right)\right), w\right\rangle$. Any element of $F^{*}$ appears to be a distribution of order one on $\bar{\Omega}$.

So the theorem of Lagrange multipliers implies the existence, at any critical point $(u, v)$, of a distribution ${ }^{5} \Lambda$ on $\bar{\Omega}$ such that, for any pair of smooth test functions $(h, k)$,

$$
\int_{\Omega}\left(\partial_{1} l \cdot h(x)+\partial_{2} l \cdot \nabla h(x)+\partial_{3} l \cdot \nabla k(x)\right) d \mathcal{H}^{3}+\langle\Lambda,(k-\nabla h)\rangle=0
$$

where the partial derivatives of $l$ are taken at $(u(x), \nabla u(x), \nabla v(x))$. Using $\mathcal{H}_{\mid \Omega}^{3}:=1_{\Omega} \mathcal{H}^{3}$, the restriction to $\Omega$ of the three-dimensional Hausdorff measure, this condition can be rewritten in terms of distributions on $\bar{\Omega}$

$$
\left\langle\partial_{1} l \mathcal{H}_{\mid \Omega}^{3}, h\right\rangle+\left\langle\partial_{2} l \mathcal{H}_{\mid \Omega}^{3}, \nabla h\right\rangle+\left\langle\partial_{3} l \mathcal{H}_{\mid \Omega}^{3}, \nabla k\right\rangle+\langle\Lambda,(k-\nabla h)\rangle=0
$$

or

$$
\left\langle\left(\partial_{1} l \mathcal{H}_{\mid \Omega}^{3}-\operatorname{div}\left(\partial_{2} l \mathcal{H}_{\mid \Omega}^{3}\right)-\operatorname{div}(\Lambda)\right), h\right\rangle+\left\langle\left(\operatorname{div}\left(\partial_{3} l \mathcal{H}_{\mid \Omega}^{3}\right)+\Lambda\right), k\right\rangle=0 .
$$

Hence, still in the sense of distributions on $\bar{\Omega}$,

$$
\partial_{1} l \mathcal{H}_{\mid \Omega}^{3}-\operatorname{div}\left(\partial_{2} l \mathcal{H}_{\mid \Omega}^{3}\right)-\operatorname{div}(\Lambda)=0, \quad \text { and } \quad \operatorname{div}\left(\partial_{3} l \mathcal{H}_{\mid \Omega}^{3}\right)+\Lambda=0 .
$$

Eliminating the Lagrange multiplier $\Lambda$ is straightforward. We get

$$
\partial_{1} l \mathcal{H}_{\mid \Omega}^{3}-\operatorname{div}\left(\partial_{2} l \mathcal{H}_{\mid \Omega}^{3}\right)+\operatorname{div}\left(\operatorname{div}\left(\partial_{3} l \mathcal{H}_{\mid \Omega}^{3}\right)\right)=0
$$

Now we make use twice of the divergence theorem (3). We obtain successively

$$
\begin{gathered}
\left(\partial_{1} l-\operatorname{div}\left(\partial_{2} l\right)\right) \mathcal{H}_{\mid \Omega}^{3}+n \cdot \partial_{2} l \mathcal{H}_{\mid \partial \Omega}^{2}+\operatorname{div}\left(\operatorname{div}\left(\partial_{3} l\right) \mathcal{H}_{\mid \Omega}^{3}\right)-\operatorname{div}\left(n \cdot \partial_{3} l \mathcal{H}_{\mid \partial \Omega}^{2}\right)=0 \\
\left(\partial_{1} l-\operatorname{div}\left(\partial_{2} l\right)+\operatorname{div}\left(\operatorname{div}\left(\partial_{3} l\right)\right)\right) \mathcal{H}_{\mid \Omega}^{3}+n \cdot\left(\partial_{2} l-\operatorname{div}\left(\partial_{3} l\right)\right) \mathcal{H}_{\mid \partial \Omega}^{2}-\operatorname{div}\left(n \cdot \partial_{3} l \mathcal{H}_{\mid \partial \Omega}^{2}\right)=0
\end{gathered}
$$

We apply now a more general version of Stokes theorem, valid on a sub-manifold with boundary ${ }^{6}$ :

$$
\operatorname{div}\left(\varphi \mathcal{H}_{\mid \partial \Omega}^{2}\right)=\operatorname{div}_{/ /}(\varphi / /) \mathcal{H}_{\mid \partial \Omega}^{2}-\tau \cdot \varphi \mathcal{H}_{\mid \partial \partial \Omega}^{1}-(n \cdot \varphi) \mathrm{D}_{\mathrm{n} \mid \partial \Omega}
$$

\footnotetext{
${ }^{5} \mathrm{~A}$ frequent error consists in introducing the Lagrange multiplier $\Lambda$ as a function. We will soon see that a part of $\Lambda$ is a measure concentrated on the boundary of the domain. Note that the constraint $v=\nabla u$ has some implications on the boundary: owing to this constraint $\nabla u$ has, like $v$, a trace on the boundary of $\Omega$ which is not natural in $H^{1}(\Omega)$.

${ }^{6}$ Indeed, for any $C^{1}$ function $\varphi$ and any smooth test function $\psi$,

$$
\begin{aligned}
\left\langle\operatorname{div}\left(\varphi \mathcal{H}_{\mid \partial \Omega}^{2}\right), \psi\right\rangle & =-\left\langle\varphi \mathcal{H}_{\mid \partial \Omega}^{2}, \nabla \psi\right\rangle=-\int_{\partial \Omega} \varphi \cdot \nabla \psi d \mathcal{H}^{2} \\
& =-\int_{\partial \Omega}(n \cdot \varphi)(n \cdot \nabla \psi) d \mathcal{H}^{2}-\int_{\partial \Omega} \varphi / / \cdot \nabla_{/ /} \psi d \mathcal{H}^{2} \\
& =-\int_{\partial \Omega}(n \cdot \varphi)(n \cdot \nabla \psi) d \mathcal{H}^{2} \\
& +\int_{\partial \Omega} \operatorname{div}_{/ /}(\varphi / /) \psi d \mathcal{H}^{2}-\int_{\partial \partial \Omega}\left(\tau \cdot \varphi_{/ /}\right) \psi d \mathcal{H}^{1} .
\end{aligned}
$$
}


where $\tau$ stands for the vector tangent to the sub-manifold and normal to its boundary, $\varphi_{/ /}$for the projection of $\varphi$ onto the tangent space, div// for the tangential divergence operator and $\mathrm{D}_{\mathrm{n} \mid \partial \Omega}$ for the normal derivative distribution. We obtain, by using this theorem on all faces (i.e. $C^{1}$ parts) of the boundary ${ }^{7}$,

$$
\begin{aligned}
& \left(\partial_{1} l-\operatorname{div}\left(\partial_{2} l\right)+\operatorname{div}\left(\operatorname{div}\left(\partial_{3} l\right)\right)\right) \mathcal{H}_{\mid \Omega}^{3}-\left(n \cdot\left(n \cdot \partial_{3} l\right)\right) \mathrm{D}_{\mathrm{n} \mid \partial \Omega} \\
& \left.\quad+n \cdot\left(\partial_{2} l-\operatorname{div}\left(\partial_{3} l\right)-\operatorname{div}_{/ /}\left(\left(n \cdot \partial_{3} l\right)\right)_{/ /}\right)\right) \mathcal{H}_{\mid \partial \Omega}^{2}-\llbracket \tau \cdot\left(n \cdot \partial_{3} l\right) \rrbracket \mathcal{H}_{\mid \partial \partial \Omega}^{1}=0 .
\end{aligned}
$$

As the distributions $\mathcal{H}_{\mid \Omega}^{3}, \mathcal{H}_{\mid \partial \Omega}^{2}, \mathcal{H}_{\mid \partial \partial \Omega}^{1}, \mathrm{D}_{\mathrm{n} \mid \partial \Omega}$ are orthogonal [7], we deduce the four criticality conditions,

$$
\begin{aligned}
& \left(\partial_{1} l-\operatorname{div}\left(\partial_{2} l\right)+\operatorname{div}\left(\operatorname{div}\left(\partial_{3} l\right)\right)\right)=0 \quad \mathcal{H}^{3} \text { a.e. in } \Omega, \\
& n \cdot\left(\partial_{2} l-\operatorname{div}\left(\partial_{3} l\right)-\operatorname{div} / /\left(\left(n \cdot \partial_{3} l\right) / /\right)\right)=0 \quad \mathcal{H}^{2} \text { a.e. on } \partial \Omega, \\
& \llbracket \tau \cdot\left(n \cdot \partial_{3} l\right) \rrbracket=0 \quad \mathcal{H}^{1} \text { a.e. on } \partial \partial \Omega, \\
& n \cdot\left(n \cdot \partial_{3} l\right)=0 \quad \mathcal{H}^{2} \text { a.e. on } \partial \Omega .
\end{aligned}
$$

Of course these conditions can be made non homogeneous by adding some boundary terms in the Lagrangian. If we remind that constraint $C(u, v)=0$ enables us to eliminate the variable $v$, these conditions correspond to the classical conditions (respectively: body force, surface boundary force, edge force, surface boundary double force) obtained by studying directly the second gradient Lagrangian $[12,13]$.

Note that the expression of the Lagrange multiplier is explicit. We have $\Lambda=-\operatorname{div}\left(\partial_{3} l \mathcal{H}_{\mid \Omega}^{3}\right)$ which becomes, by using (3),

$$
\Lambda=-\operatorname{div}\left(\partial_{3} l\right) \mathcal{H}_{\mid \Omega}^{3}+\left(n \cdot \partial_{3} l\right) \mathcal{H}_{\mid \partial \Omega}^{2} .
$$

It is now clear that $\Lambda$ involves both a volume part and a boundary part. Assuming that $\Lambda$ is a function generally leads to wrong results.

Note also that a seemingly natural idea could be to write the constraint under the form

$$
\int_{\Omega}\|v-\nabla u\|^{2} d \mathcal{H}^{3}=0
$$

and to infer from the fact that this constraint takes values in $\mathbb{R}$ the existence of a scalar Lagrange multiplier. A straightforward computation would again lead to equation (5) but with $\Lambda=2 \lambda(v-\nabla u)$. This is in complete contradiction with the previous remark. The reader must be aware that such a way of treating the constraint is erroneous as the function $(u, v) \rightarrow \int_{\Omega}\|v-\nabla u\|^{2} d \mathcal{H}^{3}=0$ is not a submersion (see entry [1]).

\footnotetext{
${ }^{7}$ Each edge in $\partial \partial \Omega$ is bordered by two faces. Thus the application of Stokes theorem on these two faces leads to two contributions on the edge involving the normals $n_{1}$ and $n_{2}$ of these faces and the vectors $\tau_{1}$ and $\tau_{2}$ tangent to the faces and normal to the edge. Notation $\llbracket \tau \cdot\left(n \cdot \partial_{3} l\right) \rrbracket$ stands for $\tau_{1} \cdot\left(n_{1} \cdot \partial_{3} l\right)+\tau_{2} \cdot\left(n_{2} \cdot \partial_{3} l\right)$.
} 


\subsection{Approach through penalization}

An intuitive way for dealing with constrained problems is to penalize the fact that the constraint is not verified. Looking for minimizers of the total energy, one decides to look for a sequence of approximate minimizers by considering, instead of the original potential subjected to the constraint, the original Lagrangian to which one adds a penalization term: for instance

$$
\mathcal{L}_{\varepsilon}(u, v)=\mathcal{L}(u, v)+\varepsilon^{-1} \int_{\Omega}\|C(u, v)\|^{2} d \mathcal{H}^{3} .
$$

Denoting $\mu_{\varepsilon}:=2 \varepsilon^{-1} C(u, v)$, any critical pair $(u, v)$ satisfies

$$
\int_{\Omega}\left(\partial_{1} l \cdot h+\partial_{2} l \cdot k+\partial_{3} l \cdot \nabla h+\partial_{4} l \cdot \nabla k+\mu_{\varepsilon} \cdot(k-\nabla h)\right) d \mathcal{H}^{3}=0
$$

which is exactly the same equation as equation (5) where the measure $\Lambda_{\varepsilon}:=$ $\mu_{\varepsilon} \mathcal{H}_{\mid \Omega}^{3}$ replaces the distribution $\Lambda$. The criticality conditions still read

$$
\begin{aligned}
& \partial_{1} \ell \mathcal{H}_{\mid \Omega}^{3}-\operatorname{div}\left(\partial_{3} \ell \mathcal{H}_{\mid \Omega}^{3}\right)+\operatorname{div}\left(\mu_{\varepsilon} \mathcal{H}_{\mid \Omega}^{3}\right)=0, \\
& \partial_{2} \ell \mathcal{H}_{\mid \Omega}^{3}-\operatorname{div}\left(\partial_{4} \ell \mathcal{H}_{\mid \Omega}^{3}\right)+\mu_{\varepsilon} \mathcal{H}_{\mid \Omega}^{3}=0
\end{aligned}
$$

or equivalently

$$
\begin{aligned}
& \partial_{1} \ell \mathcal{H}_{\mid \Omega}^{3}-\operatorname{div}\left(\left(\partial_{2} \ell+\partial_{3} \ell\right) \mathcal{H}_{\mid \Omega}^{3}\right)+\operatorname{div}\left(\operatorname{div}\left(\partial_{4} \ell \mathcal{H}_{\mid \Omega}^{3}\right)\right)=0, \\
& \partial_{2} \ell \mathcal{H}_{\mid \Omega}^{3}-\operatorname{div}\left(\partial_{4} \ell \mathcal{H}_{\mid \Omega}^{3}\right)+\mu_{\varepsilon} \mathcal{H}_{\mid \Omega}^{3}=0,
\end{aligned}
$$

Using (3), equation (13) can be rewritten

$$
\left(\partial_{2} \ell+\operatorname{div}\left(\partial_{4} \ell\right)+\mu_{\varepsilon}\right) \mathcal{H}_{\mid \Omega}^{3}-\left(n \cdot \partial_{4} \ell\right) \mathcal{H}_{\mid \partial \Omega}^{2}=0
$$

which leads to

$$
\begin{aligned}
& \partial_{2} \ell+\operatorname{div}\left(\partial_{4} \ell\right)+\mu_{\varepsilon}=0 \quad \mathcal{H}^{3} \text { a.e. in } \Omega, \\
& n \cdot \partial_{4} \ell=0 \quad \mathcal{H}^{2} \text { a.e. on } \partial \Omega .
\end{aligned}
$$

Even if equation (12) has been already found and treated in the previous section, let us do it again taking into account (16). Using (3), taking into account (16) and using again (3), we get

$$
\begin{aligned}
0 & =\partial_{1} \ell \mathcal{H}_{\mid \Omega}^{3}-\operatorname{div}\left(\left(\partial_{2} \ell+\partial_{3} \ell\right) \mathcal{H}_{\mid \Omega}^{3}\right)+\operatorname{div}\left(\operatorname{div}\left(\partial_{4} \ell\right) \mathcal{H}_{\mid \Omega}^{3}-n \cdot \partial_{4} \ell \mathcal{H}_{\mid \partial \Omega}^{2}\right)= \\
& =\partial_{1} \ell \mathcal{H}_{\mid \Omega}^{3}-\operatorname{div}\left(\left(\partial_{2} \ell+\partial_{3} \ell-\operatorname{div}\left(\partial_{4} \ell\right)\right) \mathcal{H}_{\mid \Omega}^{3}\right)= \\
& =\left(\partial_{1} \ell-\operatorname{div}\left(\partial_{2} \ell+\partial_{3} \ell-\operatorname{div}\left(\partial_{4} \ell\right)\right)\right) \mathcal{H}_{\mid \Omega}^{3}-n \cdot\left(\partial_{2} \ell+\partial_{3} \ell-\operatorname{div}\left(\partial_{4} \ell\right)\right) \mathcal{H}_{\mid \partial \Omega}^{2} .
\end{aligned}
$$

which leads to

$$
\begin{aligned}
& \partial_{1} \ell-\operatorname{div}\left(\partial_{2} \ell+\partial_{3} \ell-\operatorname{div}\left(\partial_{4} \ell\right)\right)=0 \quad \mathcal{H}^{3} \text { a.e. in } \Omega, \\
& n \cdot\left(\partial_{2} \ell+\partial_{3} \ell-\operatorname{div}\left(\partial_{4} \ell\right)\right)=0 \quad \mathcal{H}^{2} \text { a.e. on } \partial \Omega .
\end{aligned}
$$


Recalling the relationship linking $l$ and $\ell$, bulk equilibrium equation (17) is identical to (6) while boundary force balance (18) is identical to (7). However equation (16) is much stronger than (9) and there is no more edge equation corresponding to (8). This discrepancy makes dangerous to interpret second gradient boundary conditions starting from a micromorphic model. The point is that one does not know how to pass to the limit as $\varepsilon$ tends to zero. The considered penalization is a singular perturbation: we know from our previous study that equation (16), valid for any $\varepsilon>0$, is no more valid in the limit. This comes from the fact that the function $\Lambda_{\varepsilon}$ converges to a measure partially concentrated on the boundary. Hence the way we decomposed distribution (14) is not valid in the limit.

\section{References}

[1] dell'Isola F., Di Cosmo F. (2018) Lagrange Multipliers in InfiniteDimensional Systems, Methods of. In: Altenbach H., Öchsner A. (eds) Encyclopedia of Continuum Mechanics. Springer, Berlin, Heidelberg

[2] Glüge R. (2018) Continuum Mechanics Basics, Introduction and Notations. In: Altenbach H., Öchsner A. (eds) Encyclopedia of Continuum Mechanics. Springer, Berlin, Heidelberg

[3] Lagrange, Joseph Louis. Mécanique analytique. Vol. 1. Mallet-Bachelier, 1853.

[4] Dautray, Robert, and Jacques-Louis Lions. Mathematical analysis and numerical methods for science and technology: volume 3 spectral theory and applications. Springer Science \& Business Media, 2012.

[5] Rudin, Walter. Real and complex analysis. McGraw-Hill, 1987.

[6] Schweizer, Ben. On Friedrichs inequality, Helmholtz decomposition, vector potentials, and the div-curl lemma. Springer International Publishing, 2018, p. 65-79.

[7] Schwartz, Laurent, Théorie des distributions. Vol. 2. Paris: Hermann, 1957.

[8] Forest, Samuel, Cordero, N. M., Busso, Esteban P. First vs. second gradient of strain theory for capillarity effects in an elastic fluid at small length scales. Computational Materials Science, 2011, vol. 50, no 4, p. 1299-1304.

[9] dell'Isola, Francesco, Madeo, Angela, Seppecher, Pierre, How contact interactions may depend on the shape of Cauchy cuts in Nth gradient continua: approach "à la D'Alembert", Zeitschrift fur angewandte Mathematik und Physik, 2012, vol. 63, no 6, p. 1119-1141.

[10] dell'Isola, Francesco, Madeo, Angela, Seppecher, Pierre, Cauchy tetrahedron argument applied to higher contact interactions. Archive for Rational Mechanics and Analysis, 2016, vol. 219, no 3, p. 1305-1341. 
[11] Della Corte, Alessandro, dell'Isola, Francesco, Seppecher, Pierre. The postulations à la D'Alembert and à la Cauchy for higher gradient continuum theories are equivalent: a review of existing results. Proceedings of the Royal Society A: Mathematical, Physical and Engineering Sciences, 2015, vol. 471, no 2183 , p. 20150415.

[12] Mindlin, Raymond David, Micro-structure in linear elasticity, Archive for Rational Mechanics and Analysis, 1964, vol. 16, no 1, p. 51-78.

[13] Germain, Paul, La méthode des puissances virtuelles en mécanique des milieux continus, J. Mécanique, 1973, vol. 12, p. 236-274. 